\title{
Averaging Method for Neutral Stochastic Delay Differential Equations Driven by Fractional Brownian Motion
}

\author{
Peiguang Wang (iD) and Yan Xu \\ College of Mathematics and Information Science, Hebei University, Baoding, 071002 Hebei, China \\ Correspondence should be addressed to Yan Xu; xuyanbd@hbu.edu.cn
}

Received 30 March 2020; Accepted 1 May 2020; Published 29 May 2020

Guest Editor: Chuanjun Chen

Copyright (C) 2020 Peiguang Wang and Yan Xu. This is an open access article distributed under the Creative Commons Attribution License, which permits unrestricted use, distribution, and reproduction in any medium, provided the original work is properly cited.

In this paper, we investigate the stochastic averaging method for neutral stochastic delay differential equations driven by fractional Brownian motion with Hurst parameter $H \in(1 / 2,1)$. By using the linear operator theory and the pathwise approach, we show that the solutions of neutral stochastic delay differential equations converge to the solutions of the corresponding averaged stochastic delay differential equations. At last, an example is provided to illustrate the applications of the proposed results.

\section{Introduction}

Since Kolmogorov's work began in 1940 [1], the fractional Brownian motion $\left\{B^{H}(t), t \geq 0\right\}$ with Hurst parameter $H$ $\epsilon(0,1)$ has been studied by some authors $[2,3] . B^{H}(t)$ is a zero mean Gaussian stochastic process with a covariance function determined by parameter $H$. It is selfsimilar and has the stationary increments and its increment process has long-range dependence when Hurst parameter $1 / 2<H<1$, which makes $B^{H}(t)$ a suitable candidate to model many complex phenomena in finance and other practical problems. When $H \neq 1 / 2$, the $B^{H}(t)$ neither is a semimartingale nor a Markov process. These properties mean that the analysis tools for the classical stochastic differential equation theory no longer work. The most obvious problem is how to define a proper notion of stochastic integral with respect to $B^{H}(t)$. Three main integration techniques with regard to $B^{H}(t)$ have been researched, see, for example, [4-8] and the references therein.

In recent years, there has been much interest in a stochastic averaging method, which provides a powerful tool to approximate the original dynamical systems under random fluctuations by a simpler system. The first work, which was introduced by Khasminskii [9], investigated a stochastic averaging method for stochastic differential equations with Gaussian random fluctuations. Since then, the stochastic averaging method has been developed for many types of stochastic differential equations, see, e.g., [10-15].

Some phenomena of biological dynamics, engineering, and financial markets can be better understood when the effect of time delays is considered in the models, see, e.g., [16]. Hence, stochastic delay differential equations driven by fractional Brownian motion are proposed and have recently attracted great attention [17-19]. However, except [20] studied averaging method for stochastic delay differential equations of neutral type driven by G-Brownian motion, the averaging method for neutral stochastic delay differential equations is seldom considered. In this paper, our aim is to study the stochastic averaging method for neutral stochastic delay differential equations driven by fractional Brownian motion.

\section{Model Description and Preliminaries}

The aim of this section is to introduce the model and some preliminary lemmas.

The following notations are needed in this paper.

Let $\left(\Omega, F,\left\{F_{t}\right\}_{t \geq 0}, P\right)$ be a complete probability space. $B^{H}(t)$ is a $m$-dimensional fractional Brownian motion with Hurst parameter $H \in(1 / 2,1)$ defined on the space 
$\left(\Omega, F,\left\{F_{t}\right\}_{t \geq 0}, P\right)$. Let $|\bullet|$ denote the Euclidean norm. Let $\mathbb{C}=C\left((-\infty, 0] ; \mathbb{R}^{d}\right)$ denote the family of all continuous functions $\xi:(-\infty, 0] \longrightarrow \mathbb{R}^{d}$ with the norm $\|\xi\|_{\mathbb{C}}=\sup _{-\infty<s \leq 0}$ $|\xi(s)|$. For a stochastic process $\xi=\{\xi(t), t \in(-\infty, T]\}, \xi_{t}$ $\in \mathbb{C}$ defined by $\xi_{t}(s)=\xi(t+s), s \in(-\infty, 0], t \in[0, T]$. Throughout the paper, the symbol $N$ stands for a generic constant, whose value can change from one line to another.

In this paper, we will discuss the following neutral stochastic delay differential equations driven by fractional Brownian motion:

$$
\begin{aligned}
d[x(t)+c(t) x(t-\tau(t))]= & f(t, x(t), x(t-\tau(t))) d t \\
& +g(t, x(t), x(t-\tau(t))) d B^{H}(t)
\end{aligned}
$$

with initial condition $x_{0}=\xi \in C\left((-\infty, 0] ; \mathbb{R}^{d}\right)$, Where $c \in C$ $((-\infty, T],[0,1)), 0<T<\infty$. The function $\tau:[0, T] \longrightarrow \mathbb{R}^{+}$ is the time delay. The mappings $f=\left(f^{i}\right)_{d \times 1}, f^{i}:[0, T] \times$ $\mathbb{R}^{d} \times \mathbb{R}^{d} \longrightarrow \mathbb{R}, \quad g=\left(g^{i, j}\right)_{d \times m}, g^{i, j}:[0, T] \times \mathbb{R}^{d} \times \mathbb{R}^{d} \longrightarrow \mathbb{R}$ are Borel measurable.

The following operator theory is useful to obtain our main results.

Lemma 1 (see [21]). Suppose that D is a bounded linear operator on Banach space $X$, if $\|D\|<1$, then $I-D$ has a bounded inverse operator $(I-D)^{-1}$, and $\left\|(I-D)^{-1}\right\| \leq 1 /(1-\|D\|)$.

Lemma 2 (see [21]). Assume that D is a bounded linear operator on Banach space $X$ and has an inverse bounded operator, for arbitrary $\Delta D: X \longrightarrow X$, if $\|\Delta D\|<1 /\left\|D^{-1}\right\|$, then $S=D+$ $\Delta D$ has a bounded inverse, and $S^{-1}=\sum_{j=0}^{\infty}(-1)^{j} D^{-1}$ $\left(D^{-1} \Delta D\right)^{j}$.

Let the Banach space

$$
Q=\left\{x:\left.x\right|_{(-\infty, T]} \in C\left((-\infty, 0] ; \mathbb{R}^{d}\right)\right\}
$$

$\Phi: Q \longrightarrow Q$ be defined by

$$
(\Phi x)(t)=x(t)+c(t) x(t-\tau(t)) .
$$

Lemma 3. Let $c^{M}=\max _{t \in(-\infty, T]} c(t)$. If $0 \leq c(t)<1$, then $\Phi$ has a bounded inverse $\Phi^{-1}$ on $Q$, and for all $x \in Q$

$$
\left(\Phi^{-1} x\right)(t)=\sum_{j \geq 0} \prod_{0 \leq i \leq j-1}(-1)^{j} c(t-i \tau(t)) x(t-j \tau(t))
$$

$\left\|\Phi^{-1} x\right\| \leq\|x\| / 1-c^{M}$.

Proof. The proof processes are similar to Lemma 2.6 in [22], so we omit it here.
We note that $\Phi$ and $\Phi^{-1}$ are both linear operators. Then, the system (1) can be changed by the inverse transformation of $\Phi$ into the following:

$$
\begin{aligned}
d u(t)= & f\left(t,\left(\Phi^{-1} u\right)(t),\left(\Phi^{-1} u\right)(t-\tau(t))\right) d t \\
& +g\left(t,\left(\Phi^{-1} u\right)(t),\left(\Phi^{-1} u\right)(t-\tau(t))\right) d B^{H}(t)
\end{aligned}
$$

with initial condition $u_{0}=\left(\Phi^{-1} x_{0}\right)$. So, we can conclude that $u(t)$ is a solution of system (5) if and only if $\left(\Phi^{-1} u\right)(t)$ is a solution of system (1).

System (5) can be written in the integral form:

$$
\begin{aligned}
u(t)= & u_{0}+\int_{0}^{t} f\left(s,\left(\Phi^{-1} u\right)(s),\left(\Phi^{-1} u\right)(s-\tau(s))\right) d s \\
& +\int_{0}^{t} g\left(s,\left(\Phi^{-1} u\right)(s),\left(\Phi^{-1} u\right)(s-\tau(s))\right) d B^{H}(s)
\end{aligned}
$$

where $\int_{0}^{t} g(s, x(s), x,(s-\tau(s))) d B^{H}(s)$ is a pathwise-defined integral which can be represented by fractional derivatives, see, e.g., Young et al. [4], Zähle [5], and Nualart and Răşcanu [7].

Let $H \in(1 / 2,1), 1-H<\alpha<1 / 2$, denoted by $W_{(-\infty, T]}^{\alpha, \infty}$, the space of measurable functions $h:(-\infty, T] \longrightarrow R^{d}$ with the norm

$$
\|h\|_{t}=\|h\|_{\infty, t}+\int_{0}^{t}\left\|h \cdot_{+t-s}-h \cdot\right\|_{\infty, s}(t-s)^{-(\alpha+1)} d s<\infty,
$$

where $\|h\|_{\infty, t}=\sup _{-\infty<s \leq t}|h(s)|$.

Let $\alpha \in(0,1 / 2)$ be fixed. Denoting $W_{0}^{\alpha, 1}$ the space of measurable functions $h:[0, T] \longrightarrow \mathbb{R}^{d}$ such that

$$
\|h\|_{\alpha, 1}:=\int_{0}^{T} \frac{|h(s)|}{s^{\alpha}} d s+\int_{0}^{T} \int_{0}^{s} \frac{|h(s)-h(y)|}{(s-y)^{\alpha+1}} d y d s<\infty .
$$

For $h \in W_{0}^{\alpha, 1}$, defining the left-side fractional derivative $D_{0+}^{\alpha} h(u)$ by

$$
D_{0+}^{\alpha} h(u)=\frac{1}{\Gamma(1-\alpha)}\left[\frac{h(u)}{u^{\alpha}}+\alpha \int_{0}^{u} \frac{h(u)-h(y)}{(u-y)^{1+\alpha}} d y\right] 1_{(0, T)}(u) .
$$

Also denoted by $W_{T}^{1-\alpha, \infty}$ is the space of measurable functions $h:[0, T] \longrightarrow \mathbb{R}^{m}$ such that

$\|h\|_{1-\alpha, \infty, T}:=\sup _{0 \leq s<t \leq T}\left(\frac{|h(t)-h(s)|}{(t-s)^{1-\alpha}}+\int_{s}^{t} \frac{|h(y)-h(s)|}{(y-s)^{2-\alpha}} d y\right)<\infty$. 
For any $h \in W_{T}^{1-\alpha, \infty}$, setting $h_{t-}(u):=h(u)-h(t)$ and defining the right-sided fractional derivative $D_{t-}^{1-\alpha} h_{t-}(u)$ by

$$
\begin{aligned}
D_{t-}^{1-\alpha} h_{t-}(u)= & \frac{(-1)^{1-\alpha}}{\Gamma(\alpha)}\left[\frac{h(u)-h(t)}{(t-u)^{1-\alpha}}\right. \\
& \left.+(1-\alpha) \int_{u}^{t} \frac{h(u)-h(y)}{(y-u)^{2-\alpha}} d y\right] 1_{(0, t)}(u) .
\end{aligned}
$$

For $z \in W_{T}^{1-\alpha, \infty}$, we have

$$
\begin{aligned}
\Lambda_{\alpha}(z) & :=\frac{1}{\Gamma(1-\alpha)} \sup _{0<s<t<T}\left|\left(D_{t-}^{1-\alpha} z_{t-}\right)(s)\right| \\
& \leq \frac{1}{\Gamma(1-\alpha) \Gamma(\alpha)}\|z\|_{1-\alpha, \infty, T}<\infty,
\end{aligned}
$$

where $\Gamma(\cdot)$ is the gamma function. Moreover, if $h \in W_{0}^{\alpha, 1}$ and $z \in W_{T}^{1-\alpha, \infty}$, one can define the integral $\int_{0}^{t} h d z$ in the sense of Zähle [5] for all $t \in[0, T]$ and it follows from Nualart and Răşcanu [7] that

$$
\left|\int_{0}^{t} h d z\right| \leq \Lambda_{\alpha}(z)\|h\|_{\alpha, 1}
$$

For our purpose, we adopt the following hypotheses on the coefficients.

$\left(H_{1}\right)$ The function $g(t, x, y)$ is continuously differentiable in variables $x$ and $y$ and there exist some constants $\beta, \delta, 0<\beta, \delta \leq 1$ such that the following properties hold for all $x, y, \tilde{x}, \tilde{y} \in \mathbb{R}^{d}, t \in[0, T]$ :

$$
\begin{aligned}
|g(t, x, y)-g(t, \tilde{x}, \tilde{y})| \leq & N(|x-\tilde{x}|+|y-\tilde{y}|),\left|\partial_{x_{i}} g(t, x, y)-\partial_{x_{i}} g(t, \tilde{x}, y)\right|+\left|\partial_{y_{i}} g(t, x, y)-\partial_{y_{i}} g(t, x, \tilde{y})\right| \\
\leq & N\left(|x-\tilde{x}|^{\delta}+|y-\tilde{y}|^{\delta}\right),|g(t, x, y)-g(s, x, y)|+\left|\partial_{x_{i}} g(t, x, y)-\partial_{x_{i}} g(s, x, y)\right| \\
& +\left|\partial_{y_{i}} g(t, x, y)-\partial_{y_{i}} g(s, x, y)\right| \leq N|t-s|^{\beta},\left|\partial_{x_{i}} g(t, x, y)\right|+\left|\partial_{y_{i}} g(t, x, y)\right| \\
\leq & N,|g(t, x, y)| \leq N(1+|x|+|y|) .
\end{aligned}
$$

$\left(H_{2}\right)$ The function $f(t, x, y)$ is Lipschitz continuous and has linear growth in the variable $x$ and $y$, uniformly in $t \in[0, T]$, that is, $|f(t, x, y)-f(t, \tilde{x}, \tilde{y})| \leq N(|x-\tilde{x}|+|y-\tilde{y}|)$, $|f(t, x, y)| \leq N(1+|x|+|y|)$.

Let $\alpha_{0}=\min \{1 / 2, \beta, \delta /(1+\delta)\}$. Under the hypotheses $\left(H_{1}\right)-\left(H_{2}\right)$ with $\beta>1-H$ and $\delta>(1 / H)-1$, we can follow the work of Shevchenko [8] to testify that system (5) has a unique solution $u(t) \in W_{(-\infty, T]}^{\alpha, \infty}$, for all $\alpha \in\left(1-H, \alpha_{0}\right)$. Hence, system (1) has a unique solution $\left(\Phi^{-1} u\right)(t)=x(t) \in W_{(-\infty, T]}^{\alpha, \infty}$. Furthermore, we can deduce $g \in W_{0}^{\alpha, 1}$ according to Nualart and Răşcanu [7]. On the other hand, the trajectories of $B^{H}$ are almost surely locally $\beta^{\prime}$-Hölder-continuous for all $0<\beta^{\prime}<H$ see Zähle [5]. Then, $B^{H}$ belongs to the space $W_{T}^{1-\alpha, \infty}$. Hence, from the above discussion, we have the following estimate:

$$
\left|\int_{0}^{t} g(s) d B^{H}(s)\right| \leq \Lambda_{\alpha}\left(B^{H}\right)\|g\|_{\alpha, 1},
$$

where $\Lambda_{\alpha}\left(B^{H}\right)$ has moments of all orders.

Given that $\epsilon \in\left[0, \epsilon_{0}\right]$ with $\epsilon_{0}>0$ is a fixed number, consider the following standard form of system (5)

$$
\begin{aligned}
d u_{\epsilon}(t)= & \epsilon f\left(t,\left(\Phi^{-1} u\right)_{\epsilon}(t),\left(\Phi^{-1} u\right)_{\epsilon}(t-\tau(t))\right) d t \\
& +\epsilon^{H} g\left(t,\left(\Phi^{-1} u\right)_{\epsilon}(t),\left(\Phi^{-1} u\right)_{\epsilon}(t-\tau(t))\right) d B^{H}(t),
\end{aligned}
$$

with $u_{\epsilon}(0)=u_{0}$. Correspondingly, the standard form of system (1) is

$$
\begin{gathered}
d\left[x_{\epsilon}(t)+c(t) x_{\epsilon}(t-\tau(t))\right]=\epsilon f\left(t, x_{\epsilon}(t), x_{\epsilon}(t-\tau(t))\right) d t \\
+\epsilon^{H} g\left(t, x_{\epsilon}(t), x_{\epsilon}(t-\tau(t))\right) d B^{H}(t),
\end{gathered}
$$

with $x_{\epsilon}(0)=x_{0}$.

Let $\bar{f}(x, y): \mathbb{R}^{d} \times \mathbb{R}^{d} \rightarrow \mathbb{R}^{d}$ and $\bar{g}(x, y): R^{d} \times R^{d} \longrightarrow$ $R^{(d \times m)}$ be measurable functions and satisfy the conditions in $\left(H_{1}\right)-\left(H_{2}\right)$ and the following inequalities:

$$
\begin{aligned}
& \left(I_{1}\right) \frac{1}{\tilde{T}} \int_{0}^{\tilde{T}}|f(s, x, y)-\tilde{f}(x, y)|^{2} d s \leq \psi_{1}(\tilde{T})\left(1+|x|^{2}+|y|^{2}\right), \\
& \left(I_{2}\right) \frac{1}{\tilde{T}} \int_{0}^{\tilde{T}}|g(s, x, y)-\tilde{g}(x, y)|^{2} d s \leq \psi_{2}(\tilde{T})\left(1+|x|^{2}+|y|^{2}\right),
\end{aligned}
$$

where $\tilde{T} \in[0, T], \psi_{i}(\tilde{T})$ are positive bounded functions with $\sup _{0 \leq \tilde{T} \leq T}\left|\psi_{i}(\tilde{T})\right| \leq N, \lim _{\tilde{T} \rightarrow \infty} \psi_{i}(\tilde{T})=0, i=1,2$. 
Let $w_{\epsilon}(t)$ denote the solution to the following averaged system:

$$
\begin{aligned}
d w_{\epsilon}(t)= & \epsilon \bar{f}\left(\left(\Phi^{-1} w\right)_{\epsilon}(t),\left(\Phi^{-1} w\right)_{\epsilon}(t-\tau(t))\right) d t \\
& +\epsilon^{H} \bar{g}\left(\left(\Phi^{-1} w\right)_{\epsilon}(t),\left(\Phi^{-1} w\right)_{\epsilon}(t-\tau(t))\right) d B^{H}(t)
\end{aligned}
$$

with $w_{\epsilon}(0)=u_{0}$. Then, $y_{\epsilon}(t):=\left(\Phi^{-1} w\right)_{\epsilon}(t)$ is the solution to the following averaged system:

$$
\begin{aligned}
d y_{\epsilon}(t)= & \epsilon \bar{f}\left(\left(\Phi^{-1} y\right)_{\epsilon}(t),\left(\Phi^{-1} y\right)_{\epsilon}(t-\tau(t))\right) d t \\
& +\epsilon^{H} \bar{g}\left(\left(\Phi^{-1} y\right)_{\epsilon}(t),\left(\Phi^{-1} y\right)_{\epsilon}(t-\tau(t))\right) d B^{H}(t)
\end{aligned}
$$

with $y_{\epsilon}(0)=x_{0}$.

Now, from the above hypotheses, we are in conditions to demonstrate the relationship between $x_{\epsilon}(t)$ and $y_{\epsilon}(t)$.

\section{Main Results and Proofs}

The main goal of this section is to use the averaging principle to investigate the neutral stochastic delay differential equations driven by fractional Brownian motion. Theorem 6 shows that the solution $y_{\epsilon}(t)$ of the averaged system (20) converges to solution $x_{\epsilon}(t)$ of the original system (17) in the sense of mean square.

The following lemmas are crucial for our analysis and are proved in Shevchenko [8] and Nualart and Răşcanu [7].

Lemma 4 (see [8]). Assume that hypotheses (H1)-(H2) hold. Then, there exists a constant $G_{0}>0$, for $t \in(-\infty, T]$ such that

$$
E\left\|u_{\epsilon}\right\|_{T}^{2} \leq G_{0} .
$$

Lemma 5 (see [7]). Assume that hypotheses $\left(H_{1}\right)-\left(H_{2}\right)$ hold. Then, there exists a constant $G_{1}>0$, for $s, t \in[0, T]$, we have

$$
\left|u_{\epsilon}(t)-u_{\epsilon}(s)\right| \leq G_{1}\left(1+2|| \Phi^{-1}|||| u_{\epsilon} \|_{T}\right)|t-s|^{1-\alpha} .
$$

From Lemma 5, we can prove similarly that $\mid w_{\epsilon}(t)-$ $w_{\epsilon}(s)\left|\leq G_{1}\left(1+2|| \Phi^{-1}|||| w_{\epsilon}|| T\right)\right| t-\left.s\right|^{1-\alpha}$.
Theorem 6. Assume that hypotheses $\left(H_{1}\right)-\left(H_{2}\right)$ and $\left(I_{1}\right)$ $-\left(I_{2}\right)$ hold. For $\epsilon \in\left(0, \epsilon_{0}\right]$ is a positive small parameter with $\epsilon_{0}$ a constant, $\theta>0$, there exist constants $\epsilon_{1} \in\left(0, \epsilon_{0}\right], O>0$ such that for any $\epsilon \in\left(0, \epsilon_{1}\right], t \in\left[0, O \epsilon^{-\lambda}\right], 0<\lambda<1$, we get

$$
\sup _{0 \leq t \leq O \epsilon^{-\lambda}} E\left|u_{\epsilon}(t)-w_{\epsilon}(t)\right|^{2} \leq \theta
$$

and then, we obtain

$$
\sup _{0 \leq t \leq O \epsilon^{-\lambda}} E\left|x_{\epsilon}(t)-y_{\epsilon}(t)\right|^{2} \leq\left\|\Phi^{-1}\right\|^{2} \theta .
$$

Proof. According to systems (16) and (19), on account of the elementary inequality, we obtain

$$
\begin{aligned}
E\left|u_{\epsilon}(t)-w_{\epsilon}(t)\right|^{2} \leq & 2 \epsilon^{2} E \mid \int_{0}^{t}\left(f\left(s,\left(\Phi^{-1} u\right)_{\epsilon}(s),\left(\Phi^{-1} u\right)_{\epsilon}(s-\tau(s))\right)\right. \\
& \left.-\bar{f}\left(\left(\Phi^{-1} w\right)_{\epsilon}(s),\left(\Phi^{-1} w\right)_{\epsilon}(s-\tau(s))\right)\right)\left.d s\right|^{2} \\
& +2 \epsilon^{2 H} E \mid \int_{0}^{t}\left(g\left(s,\left(\Phi^{-1} u\right)_{\epsilon}(s),\left(\Phi^{-1} u\right)_{\epsilon}(s-\tau(s))\right)\right. \\
& \left.-\bar{g}\left(\left(\Phi^{-1} w\right)_{\epsilon}(s),\left(\Phi^{-1} w\right)_{\epsilon}(s-\tau(s))\right)\right)\left.d B^{H}(s)\right|^{2} \\
= & J_{1}(t)+J_{2}(t),
\end{aligned}
$$

where $[0, t] \subset[0, b] \subset[0, T]$. On the one hand, for term $J_{1}(t)$, we derive

$$
\begin{aligned}
J_{1}(t)= & 2 \epsilon^{2} E \mid \int_{0}^{t}\left(f\left(s,\left(\Phi^{-1} u\right)_{\epsilon}(s),\left(\Phi^{-1} u\right)_{\epsilon}(s-\tau(s))\right)\right. \\
& \left.-\bar{f}\left(\left(\Phi^{-1} w\right)_{\epsilon}(s),\left(\Phi^{-1} w\right)_{\epsilon}(s-\tau(s))\right)\right)\left.d s\right|^{2} \\
\leq & 2 b \epsilon^{2} E \int_{0}^{t} \mid f\left(s,\left(\Phi^{-1} u\right)_{\epsilon}(s),\left(\Phi^{-1} u\right)_{\epsilon}(s-\tau(s))\right) \\
& -\left.\bar{f}\left(\left(\Phi^{-1} w\right)_{\epsilon}(s),\left(\Phi^{-1} w\right)_{\epsilon}(s-\tau(s))\right)\right|^{2} d s .
\end{aligned}
$$

On the other hand, applying (15) for term $J_{2}(t)$, it follows that

$$
\begin{aligned}
J_{2}(t) \leq & 4 \epsilon^{2 H} \Lambda_{\alpha}^{2}\left(B^{H}\right) b^{1-2 \alpha} E \int_{0}^{t}\left|g\left(s,\left(\Phi^{-1} u\right)_{\epsilon}(s),\left(\Phi^{-1} u\right)_{\epsilon}(s-\tau(s))\right)-\bar{g}\left(\left(\Phi^{-1} w\right)_{\epsilon}(s),\left(\Phi^{-1} w\right)_{\epsilon}(s-\tau(s))\right)\right|^{2} d s \\
& +4 \epsilon^{2 H} \Lambda_{\alpha}^{2}\left(B^{H}\right) E\left(\int_{0}^{t} \int_{0}^{s} \frac{1}{(s-r)^{1+\alpha}} \mid g\left(s,\left(\Phi^{-1} u\right)_{\epsilon}(s),\left(\Phi^{-1} u\right)_{\epsilon}(s-\tau(s))\right)-\bar{g}\left(\left(\Phi^{-1} w\right)_{\epsilon}(s),\left(\Phi^{-1} w\right)_{\epsilon}(s-\tau(s))\right)\right. \\
& \left.-g\left(r,\left(\Phi^{-1} u\right)_{\epsilon}(r),\left(\Phi^{-1} u\right)_{\epsilon}(r-\tau(r))\right)+\bar{g}\left(\left(\Phi^{-1} w\right)_{\epsilon}(r),\left(\Phi^{-1} w\right)_{\epsilon}(r-\tau(r))\right) \mid d r d s\right)^{2}:=J_{21}(t)+J_{22}(t) .
\end{aligned}
$$


Furthermore, by $\left(H_{1}\right)$, Lemma 4 and Lemma 5 yields

$$
\begin{aligned}
J_{22}(t) \leq & 4 \epsilon^{2 H} \Lambda_{\alpha}^{2}\left(B^{H}\right) \times E\left(\int _ { 0 } ^ { t } \left(\int_{0}^{s} \frac{\left|g\left(s,\left(\Phi^{-1} u\right)_{\epsilon}(s),\left(\Phi^{-1} u\right)_{\epsilon}(s-\tau(s))\right)-g\left(r,\left(\Phi^{-1} u\right)_{\epsilon}(r),\left(\Phi^{-1} u\right)_{\epsilon}(r-\tau(r))\right)\right|}{(s-r)^{1+\alpha}} d r\right.\right. \\
& \left.\left.+\int_{0}^{s} \frac{\left|\bar{g}\left(\left(\Phi^{-1} w\right)_{\epsilon}(s),\left(\Phi^{-1} w\right)_{\epsilon}(s-\tau(s))\right)-\bar{g}\left(\left(\Phi^{-1} w\right)_{\epsilon}(r),\left(\Phi^{-1} w\right)_{\epsilon}(r-\tau(r))\right)\right|}{(s-r)^{1+\alpha}} d r\right) d s\right)^{2} \\
\leq & N \epsilon^{2 H} \Lambda_{\alpha}^{2}\left(B^{H}\right)\left[b^{2(1+\beta-\alpha)}+\left\|\Phi^{-1}\right\|^{2}\left(G_{1}^{2}\left(1+4\left\|\Phi^{-1}\right\|^{2} G_{0}\right) b^{4-4 \alpha}+G_{0} b^{2-2 \alpha}\right)\right] .
\end{aligned}
$$

Hence, under hypotheses $\left(H_{1}\right)-\left(H_{2}\right),\left(I_{1}\right)-\left(I_{2}\right)$ and Lemma 4, we get

$$
\begin{aligned}
E\left|u_{\varepsilon}(t)-w_{\varepsilon}(t)\right|^{2} \leq & 2 b \varepsilon^{2} E \int_{0}^{t}\left|f\left(s,\left(\Phi^{-1} u\right)_{\varepsilon}(s),\left(\Phi^{-1} u\right)_{\varepsilon}(s-\tau(s))\right)-\bar{f}\left(\left(\Phi^{-1} w\right)_{\varepsilon}(s),\left(\Phi^{-1} w\right)_{\varepsilon}(s-\tau(s))\right)\right|^{2} d s \\
& +4 \varepsilon^{2 H} \Lambda_{\alpha}^{2}\left(B^{H}\right) b^{1-2 \alpha} \times E \int_{0}^{t}\left|g\left(s,\left(\Phi^{-1} u\right)_{\varepsilon}(s),\left(\Phi^{-1} u\right)_{\varepsilon}(s-\tau(s))\right)-\bar{g}\left(\left(\Phi^{-1} w\right)_{\varepsilon}(s),\left(\Phi^{-1} w\right)_{\varepsilon}(s-\tau(s))\right)\right|^{2} d s \\
& +N \varepsilon^{2 H} \Lambda_{\alpha}^{2}\left(B^{H}\right)\left[b^{2(1+\beta-\alpha)}+\left\|\Phi^{-1}\right\|^{2}\left(G_{1}^{2}\left(1+4\left\|\Phi^{-1}\right\|^{2} G_{0}\right) b^{4-4 \alpha}+G_{0} b^{2-2 \alpha}\right)\right] \\
\leq & N\left(\varepsilon^{2} b+\varepsilon^{2 H} \Lambda_{\alpha}^{2}\left(B^{H}\right) b^{1-2 \alpha}\right)\left\|\Phi^{-1}\right\|^{2} E \int_{0}^{t} \sup _{0 \leq \sigma \leq s}\left|u_{\varepsilon}(\sigma)-w_{\varepsilon}(\sigma)\right|^{2} d s+N\left(\varepsilon^{2} b^{2}+\varepsilon^{2 H} \Lambda_{\alpha}^{2}\left(B^{H}\right) b^{2-2 \alpha}\right)\left(1+2\left\|\Phi^{-1}\right\|^{2} G_{0}\right) \\
& +N \varepsilon^{2 H} \Lambda_{\alpha}^{2}\left(B^{H}\right)\left[b^{2(1+\beta-\alpha)}+\left\|\Phi^{-1}\right\|^{2}\left(G_{1}^{2}\left(1+4\left\|\Phi^{-1}\right\|^{2} G_{0}\right) b^{4-4 \alpha}+G_{0} b^{2-2 \alpha}\right)\right],
\end{aligned}
$$

and so

$$
\begin{aligned}
\sup _{0 \leq t \leq b} E\left|u_{\varepsilon}(t)-w_{\varepsilon}(t)\right|^{2} \leq & N\left(\varepsilon^{2} b+\varepsilon^{2 H} \Lambda_{\alpha}^{2}\left(B^{H}\right) b^{1-2 \alpha}\right)\left\|\Phi^{-1}\right\|^{2} E \int_{0}^{b} \sup _{0 \leq \sigma \leq s}\left|u_{\varepsilon}(\sigma)-w_{\varepsilon}(\sigma)\right|^{2} d s \\
& +N\left(\varepsilon^{2} b^{2}+\varepsilon^{2 H} \Lambda_{\alpha}^{2}\left(B^{H}\right) b^{2-2 \alpha}\right)\left(1+2\left\|\Phi^{-1}\right\|^{2} G_{0}\right) \\
& +N \varepsilon^{2 H} \Lambda_{\alpha}^{2}\left(B^{H}\right)\left[b^{2(1+\beta-\alpha)}+\left\|\Phi^{-1}\right\|^{2}\left(G_{1}^{2}\left(1+4\left\|\Phi^{-1}\right\|^{2} G_{0}\right) b^{4-4 \alpha}+G_{0} b^{2-2 \alpha}\right)\right] .
\end{aligned}
$$

Then, select $\lambda \in(0,1), O>0$, such that for each $t \in[0, O$ $\left.\varepsilon^{-\lambda}\right] \subset[0, T]$, we obtain

$$
\sup _{0 \leq t \leq O \varepsilon^{-\lambda}} E\left|u_{\varepsilon}(t)-w_{\varepsilon}(t)\right|^{2} \leq K \varepsilon^{1-\lambda}
$$

$$
\begin{aligned}
K= & N \varepsilon^{\lambda-1}\left\{\left[\left(1+2\left\|\Phi^{-1}\right\|^{2} G_{0}\right)\left(\varepsilon^{2-2 \lambda} O^{2}+\varepsilon^{2 H+2 \alpha \lambda-2 \lambda} \Lambda_{\alpha}^{2}\left(B^{H}\right) O^{2-2 \alpha}\right)\right.\right. \\
& +\varepsilon^{2 H} \Lambda_{\alpha}^{2}\left(B^{H}\right)\left(\varepsilon^{2(\alpha \lambda-\lambda-\beta \lambda)} O^{2(1+\beta-\alpha)}+\left\|\Phi^{-1}\right\|^{2}\right. \\
& \left.\left.\left.\cdot\left(G_{1}^{2}\left(1+4\left\|\Phi^{-1}\right\|^{2} G_{0}\right) \varepsilon^{4 \alpha \lambda-4 \lambda} O^{4-4 \alpha}+G_{0} \varepsilon^{2 \alpha \lambda-2 \lambda} O^{2-2 \alpha}\right)\right)\right]\right\} \\
& \times \exp \left(N\left\|\Phi^{-1}\right\|^{2}\left(\varepsilon^{2-2 \lambda} O^{2}+\varepsilon^{2 H+2 \alpha \lambda-2 \lambda} \Lambda_{\alpha}^{2}\left(B^{H}\right) O^{2-2 \alpha}\right)\right) .
\end{aligned}
$$

where 
Thus, for any given number $\theta>0$, we can choose $\varepsilon_{1}$ $\epsilon\left(0, \varepsilon_{0}\right]$ such that for each $\varepsilon \in\left(0, \varepsilon_{1}\right]$ and $t \in\left[0, O \varepsilon^{-\lambda}\right] \subset[0, T]$,

$$
\sup _{0 \leq t \leq O \varepsilon^{-\lambda}} E\left|u_{\varepsilon}(t)-w_{\varepsilon}(t)\right|^{2} \leq \theta
$$

and hence,

$$
\begin{aligned}
\sup _{0 \leq t \leq O \varepsilon^{-\lambda}} E\left|x_{\varepsilon}(t)-y_{\varepsilon}(t)\right|^{2} & =\sup _{t \in\left[0, O \varepsilon^{-\lambda}\right]} E\left|\left(\Phi^{-1} u\right)_{\varepsilon}(t)-\left(\Phi^{-1} w\right)_{\varepsilon}(t)\right|^{2} \\
& \leq\left\|\Phi^{-1}\right\|^{2} \theta .
\end{aligned}
$$

The proof is therefore complete.

Finally, as an application, we consider the following example.

Example 1. Consider the neutral stochastic delay differential equations driven by fractional Brownian motion:

$$
\begin{aligned}
d\left[x_{\varepsilon}(t)+0.8 x_{\varepsilon}(t-\tau)\right]= & \varepsilon f\left(t, x_{\varepsilon}(t), x_{\varepsilon}(t-\tau)\right) d t \\
& +\varepsilon^{H} g\left(t, x_{\varepsilon}(t), x_{\varepsilon}(t-\tau)\right) d B^{H}(t),
\end{aligned}
$$

where the initial condition $x_{\varepsilon}(0)=\{\xi(s),-\infty<s \leq 0\}, E$ $\|\xi\|^{2}<\infty, \tau>0$ is a constant, $t \in[0, T]$,

$$
\begin{aligned}
& f\left(t, x_{\varepsilon}(t), x_{\varepsilon}(t-\tau)\right)=2\left(x_{\varepsilon}(t)+x_{\varepsilon}\right. \\
& \quad \cdot(t-\tau)) \cos ^{2} t, g\left(t, x_{\varepsilon}(t), x_{\varepsilon}(t-\tau)\right)=2,
\end{aligned}
$$

$B^{H}(t)$ is a fractional Brownian motion. Then,

$$
\begin{aligned}
\bar{f}\left(x_{\varepsilon}(t), x_{\varepsilon}(t-\tau)\right) & =\frac{1}{\pi} \int_{0}^{\pi} 2\left(x_{\varepsilon}(t)+x_{\varepsilon}(t-\tau)\right) \cos ^{2} t d t \\
& =x_{\varepsilon}(t)+x_{\varepsilon}(t-\tau), \bar{g}\left(x_{\varepsilon}(t), x_{\varepsilon}(t-\tau)\right)=2 .
\end{aligned}
$$

Let $\left(\Phi x_{\varepsilon}\right)(t)=x_{\varepsilon}(t)+c(t) x_{\varepsilon}(t-\tau)$. Thus, $\left(\Phi^{-1} x_{\varepsilon}\right)(t)=$ $\sum_{j \geq 0} \prod_{0 \leq i \leq j-1}(-1)^{j} c(t-i \tau) x_{\varepsilon}(t-j \tau)$. Define a new averaged stochastic differential delay equation

$$
\begin{aligned}
d y_{\varepsilon}(t)= & \varepsilon \bar{f}\left(\left(\Phi^{-1} y\right)_{\varepsilon}(t),\left(\Phi^{-1} y\right)_{\varepsilon}(t-\tau)\right) d t \\
& +\varepsilon^{H} \bar{g}\left(\left(\Phi^{-1} y\right)_{\varepsilon}(t),\left(\Phi^{-1} y\right)_{\varepsilon}(t-\tau)\right) d B^{H}(t)
\end{aligned}
$$

namely,

$$
d y_{\varepsilon}(t)=\varepsilon\left(\left(\Phi^{-1} y\right)_{\varepsilon}(t)+\left(\Phi^{-1} y\right)_{\varepsilon}(t-\tau)\right) d t+2 \varepsilon^{H} d B^{H}(t) .
$$

Obviously, when $t \in[0, \tau]$, the explicit solution of system (39) can be obtained as

$$
\begin{aligned}
y_{\varepsilon}(t)= & e^{\varepsilon t}\left\{\xi+\varepsilon \int_{0}^{t} e^{-\varepsilon s}\left(\sum_{i \geq 1}(-0.8)^{i} y_{\varepsilon}(s-i \tau)\right.\right. \\
& \left.\left.+\sum_{i \geq 0}(-0.8)^{i} y_{\varepsilon}(s-(i+1) \tau)\right) d s+2 \varepsilon^{H} \int_{0}^{t} e^{-\varepsilon s} d B^{H}(s)\right\} .
\end{aligned}
$$

When $t \in[\tau, 2 \tau]$, the explicit solution of system (2) is given by

$$
\begin{aligned}
y_{\varepsilon}(t)= & e^{\varepsilon(t-\tau)}\left\{y_{\varepsilon}(\tau)+\varepsilon \int_{\tau}^{t} e^{-\varepsilon(s-\tau)}\left(\sum_{i \geq 1}(-0.8)^{i} y_{\varepsilon}(s-i \tau)\right.\right. \\
& \left.\left.+\sum_{i \geq 0}(-0.8)^{i} y_{\varepsilon}(s-(i+1) \tau)\right) d s+2 \varepsilon^{H} \int_{\tau}^{t} e^{-\varepsilon(s-\tau)} d B^{H}(s)\right\} .
\end{aligned}
$$

Repeating the same steps, we can deduce solution $y_{\varepsilon}(t)$ on the entire interval $[0, T]$. It is easy to see that functions $f, g, \bar{f}, \bar{g}$ in systems (35) and (39) satisfy all the conditions in $\left(H_{1}\right)-\left(H_{2}\right)$ and $\left(I_{1}\right)-\left(I_{2}\right)$; therefore, Theorem 6 holds. That is,

$$
E\left|x_{\varepsilon}(t)-y_{\varepsilon}(t)\right|^{2} \leq\left\|\Phi^{-1}\right\|^{2} \theta
$$

\section{Data Availability}

Data sharing not applicable to this article as no datasets were generated or analysed during the current study.

\section{Conflicts of Interest}

The authors declare that they have no competing interests.

\section{Authors' Contributions}

All authors completed the paper together. All authors read and approved the final manuscript.

\section{Acknowledgments}

The first author is partly supported by the National Natural Science Foundation of China under Grant Nos. 11771115 and 11271106, and the second author is partly supported by the National Natural Science Foundation of China under Grant No. 11801128 and the Natural Science Foundation of Hebei Province under Grant No. A2018201109.

\section{References}

[1] A. N. Kolmogorov, "Wienersche spiralen und einige andere interessante Kurven in Hilbertschen raum, Comptes Rendus (Doklady) de l'Académie des Sciences de l'URSS," Nouvelle Série, vol. 26, pp. 115-118, 1940. 
[2] P. Lévy and M. Loeve, Processus Stochastiques et Mouvement Brownien, GauthierVillars Paris, 1965.

[3] B. B. Mandelbrot and J. W. Van Ness, "Fractional brownian motions, fractional noises and applications," SIAM Review, vol. 10, no. 4, pp. 422-437, 1968.

[4] L. C. Young et al., "An inequality of the hölder type, connected with stieltjes integration," Acta Mathematica, vol. 67, pp. 251282, 1936.

[5] M. Zähle, "Integration with respect to fractal functions and stochastic calculus. I," Probability Theory and Related Fields, vol. 111, no. 3, pp. 333-374, 1998.

[6] A. A. Ruzmaikina, "Stieltjes integrals of hölder continuous functions with applications to fractional Brownian motion," Journal of Statistical Physics, vol. 100, no. 5/6, pp. 1049-1069, 2000.

[7] A. Rascanu and A. Rascanu, "Differential equations driven by fractional Brownian motion," Collectanea Mathematica, vol. 53, no. 1, pp. 55-81, 2002.

[8] G. Shevchenko, "Mixed fractional stochastic differential equations with jumps," Stochastics, vol. 86, no. 2, pp. 203-217, 2013.

[9] R. Z. Khas'minskii, "A limit theorem for the solutions of differential equations with random right-hand sides," Theory of Probability \& Its Applications, vol. 11, no. 3, pp. 390-406, 1966.

[10] V. Radchenko, "Averaging principle for the heat equation driven by a general stochastic measure," Statistics \& Probability Letters, vol. 146, pp. 224-230, 2019.

[11] B. Pei, Y. Xu, and J.-L. Wu, "Stochastic averaging for stochastic differential equations driven by fractional brownian motion and standard brownian motion," Applied Mathematics Letters, vol. 100, article 106006, 2020.

[12] X.-M. Li, "An averaging principle for a completely integrable stochastic hamiltonian system," Nonlinearity, vol. 21, no. 4, pp. 803-822, 2008.

[13] Y. Xu, J. Duan, and W. Xu, "An averaging principle for stochastic dynamical systems with lévy noise," Physica D: Nonlinear Phenomena, vol. 240, no. 17, pp. 1395-1401, 2011.

[14] J. Xu and J. Liu, "An averaging principle for multivalued stochastic differential equations," Stochastic Analysis and Applications, vol. 32, no. 6, pp. 962-974, 2014.

[15] Y. Xu, B. Pei, and J.-L. Wu, "Stochastic averaging principle for differential equations with non-lipschitz coefficients driven by fractional brownian motion," Stochastics and Dynamics, vol. 17, no. 2, article 1750013, pp. 1-16, 2017.

[16] B. Balachandran, T. Kalmár-Nagy, and D. E. Gilsinn, Delay Differential Equations, Springer, 2009.

[17] W. Mao, S. You, X. Wu, and X. Mao, "On the averaging principle for stochastic delay differential equations with jumps," Advances in Difference Equations, vol. 2015, Article ID 70, 2015.

[18] B. Pei, Y. Xu, G. Yin, and X. Zhang, "Averaging principles for functional stochastic partial differential equations driven by a fractional Brownian motion modulated by two-time-scale markovian switching processes," Nonlinear Analysis: Hybrid Systems, vol. 27, pp. 107-124, 2018.

[19] P. T. Hong and C. T. Binh, "A note on exponential stability of non-autonomous linear stochastic differential delay equations driven by a fractional Brownian motion with Hurst index >12," Statistics \& Probability Letters, vol. 138, pp. 127-136, 2018.
[20] X. He, S. Han, and J. Tao, "Averaging principle for sdes of neutral type driven by g-brownian motion," Stochastics and Dynamics, vol. 19, no. 1, article 1950004, 2019.

[21] J. B. Conway, A Course in Functional Analysis, Vol. 96, Springer Science \& Business Media, 2013.

[22] C. Wang, Y. Li, and Y. Fei, "Three positive periodic solutions to nonlinear neutral functional differential equations with impulses and parameters on time scales," Mathematical and Computer Modelling, vol. 52, no. 9-10, pp. 1451-1462, 2010. 